Annuaire suisse de politique de développement

\title{
Téléservices au Mali : l'art du détournement
}

Jean-François L'haire, Ousmane Ly et Marie Thorndahl

\section{CpenEdition \\ Journals}

Édition électronique

URL : http://journals.openedition.org/aspd/549

DOI : 10.4000/aspd.549

ISSN : 1663-9669

Éditeur

Institut de hautes études internationales et du développement

Édition imprimée

Date de publication : 1 novembre 2003

Pagination : 85-87

ISSN : 1660-5934

\section{Référence électronique}

Jean-François L'haire, Ousmane Ly et Marie Thorndahl, « Téléservices au Mali : l'art du

détournement ", Annuaire suisse de politique de développement [En ligne], 22-2 | 2003, mis en ligne le 22 mars 2010, consulté le 08 septembre 2020. URL : http://journals.openedition.org/aspd/549; DOI : https://doi.org/10.4000/aspd.549 


\title{
Téléservices au Mali: l'art du détournement
}

\author{
Jean-François L'haire* \\ Ousmane Ly*
}

Propos recueillis par Marie Thorndahl***

R esponsable du Service des nouvelles technologies de l'information de l'Université de Genève et vieil ami de l'Afrique, Jean-François L'haire est sceptique face aux discours vantant les mérites d'Internet pour résoudre les problèmes de développement. C'est donc d'abord pour l'industrie suisse qu'il a développé des outils d'enseignement à distance. «Les collaborateurs des entreprises bénéficieront ainsi d'une formation continue, sans devoir quitter leurs bureaux», pensait-il.

Nettement plus confiant, Ousmane Ly, jeune médecin malien, croit aux vertus des technologies de l'information: "Les TIC doivent être une priorité pour l'Afrique. Elles nous permettront de sauter bien des étapes!» Dès 1999, encore étudiant, il s'entête à vouloir mettre en place un réseau de télémédecine dans son pays. Le Mali n'a cependant qu'un accès très faible au réseau mondial, l'équivalent d'une ligne ADSL pour tout le pays.

\section{Un système ingénieux et simple de téléenseignement}

Côté Université de Genève, les outils d'enseignement à distance se déve- loppent mais peinent à trouver leur public en Suisse. Les collaborateurs de l'industrie se sont avérés des étudiants peu assidus. En revanche, les écoles et universités africaines font pression pour en bénéficier. C'est au Togo, en 2000, que le système développé à Genève fait son entrée en Afrique. Il faut dire que son originalité réside dans sa simplicité d'installation et d'utilisation. Il est peu gourmand en bande passante et ne nécessite aucun logiciel particulier.

Les séminaires et conférences dispensés à l'Université de Genève peuvent ainsi être suivi via le web. L'étudiant à distance voit le professeur, l'entend, visionne ses documents électroniques en temps réel et peut lui poser des questions par écrit. L'enseignant projette ses documents électroniques dans la salle de cours, répond aux questions des étudiants et utilise une caméra de documents pour écrire sur papier blanc ou transparent. Il est également possible de montrer des photos ou des objets, ainsi que des plans affichés contre un mur.

Le scénario reproduit donc assez fidèlement une pédagogie magistrale classique, tout en permettant l'interaction et les questions-réponses. Comme il n'est pas spectaculaire, les

\footnotetext{
* Enseignant et chef du service nouvelles technologies de l'information de l'Université de Genève, Suisse.

** Docteur et coordinateur exécutif de l'ONG «Le vestibule de la santé», Mali.

*** Socio-économiste, France.
} 
étudiants ne sont pas dispersés par un show technologique au détriment des contenus et les professeurs se sentent à l'aise. Seul un micro-cravate leur rappelle que leur auditoire est subitement démultiplié.

Conception ingénieuse mais somme toute classique. «Il s'agit d'avoir l'ambition modeste! Ce qui importe est de montrer la faisabilité par la simplicité et d'éviter les éléphants blancs», explique J.-F. L'haire. «Le génie du système est qu'on puisse facilement le détourner. Ce sont ces détournements qui nous permettront de brûler des étapes du développement! » rétorque $\mathrm{O}$. Ly.

\section{Télémédecine du Sud vers le Nord}

Egalement en 2000, une première téléconsultation neurochirurgicale entre Genève et Bamako a permis de prouver que la télémédecine peut avoir du sens même si elle utilise «seulement» une combinaison d'équipements de base. A l'aide d'une webcam (pour l'image) et du téléphone (pour le son), une petite Bamakoise, opérée trois ans plus tôt en Suisse, a pu être examinée par une équipe de neurochirurgie genevoise. Cette première expérience, qui a permis d'éviter un voyage à la patiente, encourage les équipes. Parallèlement, à l'occasion de la Coupe d'Afrique des Nations 2002, le Mali augmente de manière significative sa connectivité et crée des points d'accès dans toutes les capitales régionales. $\mathrm{Ce}$ saut technologique permet de revoir les ambitions à la hausse.

En 2002, le système de téléenseignement est utilisé pour continuer les consultations de la petite Bamakoise. Les médecins peuvent ainsi échanger des masses d'informations médicotechniques - radiographies, résultats d'examens, etc. - , se parler et voir la patiente simultanément.

Le même système est aussi installé pour son usage d'origine. Une fois par mois, les cours de la Faculté de Genève sont suivis par une centaine de médecins et d'étudiants à l'Hôpital de Bamako, dans le cadre d'un programme de formation médicale continue. Plus original, un docteur malien, spécialiste de la lèpre, a inauguré le système dans le sens SudNord, en donnant un cours de médecine tropicale. Ce n'est pas la prouesse technologique qui est en jeu mais bien «l'échange de connaissances et de pratiques ». J.-F. L'haire raconte: «Le cours a failli ne pas avoir lieu parce que les Maliens avaient trop de retard. Au bout d'une demi-heure d'attente, les étudiants genevois, excédés, ont failli partir. Le minimum est de s'entendre sur l'heure. Nous avons encore des choses à améliorer!»

\section{Lutter contre la fracture interne}

«Le vrai enjeu n'est pas là, répond O. Ly. Ce qui importe est de lutter contre la fracture interne au pays. Il faut mettre l'expertise des spécialistes maliens, à $80 \%$ basés dans la capitale, à la disposition des médecins généralistes des régions!» Le serveur de diffusion de cours installé à Bamako permet aux centres régionaux de Ségou et de Tombouctou de faire appel à des spécialistes et de suivre les cours de Genève, Bamako ou ailleurs... car le réseau des producteurs de cours s'agrandit et doit bientôt compter, en plus de Genève et Bamako, Ségou, Tombouctou, Nouakchott, Dakar et Yaoundé. C'est 
finalement un réseau de télémédecine Sud-Sud-Nord qui, pas à pas, se met en place.

Financées par un premier apport du Département de l'action sociale et de la santé de la Ville de Genève, les connexions maliennes sont aujourd'hui subventionnées par la SOTELMA, l'opérateur national des télécommunications. Bien que des institutions publiques le soutiennent, le réseau de télémédecine se monte en dehors d'un cadre institutionnel figé et des programmes internationaux de lutte contre la «fracture numérique ». Il est d'abord le fruit de rencontres entre professionnels de différentes origines scientifiques et culturelles.

Loin de mises en scène spectaculaires, les TIC ont commencé à montrer leur capacité à soutenir les médecins maliens dans leur pratique. Ces premiers succès ouvrent des perspectives pour améliorer les outils, multiplier les contenus et tester de nouveaux usages. Ils permettent aussi aux autorités politiques de s'en saisir. Après les grands discours sur les potentiels d'Internet pour renouveler la coopération Nord-Sud, il en va bien sûr aussi de leur crédibilité ! 